\title{
Comparing the empirical powers of several independence tests in generalized FGM family
}

\author{
M. Zargar ${ }^{a}$, H. Jabbari ${ }^{1, a}$, M. Amini ${ }^{a}$ \\ ${ }^{a}$ Department of Statistics, Ferdowsi University of Mashhad, Iran
}

\begin{abstract}
The powers of some tests for independence hypothesis against positive (negative) quadrant dependence in generalized Farlie-Gumbel-Morgenstern distribution are compared graphically by simulation. Some of these tests are usual linear rank tests of independence. Two other possible rank tests of independence are locally most powerful rank test and a powerful nonparametric test based on the Cramér-von Mises statistic. We also evaluate the empirical power of the class of distribution-free tests proposed by Kochar and Gupta (1987) based on the asymptotic distribution of a U-statistic and the test statistic proposed by Güven and Kotz (2008) in generalized Farlie-Gumbel-Morgenstern distribution. Tests of independence are also compared for sample sizes $n=20,30,50$, empirically. Finally, we apply two examples to illustrate the results.
\end{abstract}

Keywords: Generalized Farlie-Gumbel-Morgenstern (FGM) distribution, positive and negative quadrant dependence, rank tests, tests of independence, U-statistic

\section{Introduction}

Farlie (1960), Gumbel (1960) and Morgenstern (1956) introduced a bivariate distribution with given marginals. The Pearson correlation achievable cannot exceed 0.33 in the classical Farlie-GumbelMorgenstern (FGM) distribution with absolutely continuous marginals. Huang and Kotz (1999) studied a new modification of the classical FGM distribution to increase the dependence between the underlying variables by introducing an additional parameter. Bairamov and Kotz (2002) introduced the generalized FGM family which allows us to achieve a correlation between the components greater than 0.5. Baker (2008) proposed an extension to the FGM distribution. Dou et al. (2016) estimated parameters of Baker's (2008) distribution using EM algorithms. Some applications of FGM distribution have been recently introduced in the literature. For example, Jung et al. (2008) presented an application of generalized FGM copula function in exchange markets using directional dependence concept. Hlubinka and Kotz (2010) used the generalized FGM distribution and related copulas as bivariate models for the distribution of spheroidal characteristics. Amini et al. (2010) offered an example of FGM distribution for modelling the dependence structure of the lifetimes of two components of CPU (central processing unit) in desktop computer.

One of the most important concepts of dependence is quadrant dependence. This concept was introduced by Lehmann (1966). Let $(X, Y)$ be an absolutely continuous random vector with distribution function $H(x, y)$ and marginal distribution functions $F(x)$ and $G(y)$. We say that the pair $(X, Y)$ is positively (negatively) quadrant dependent if, $H(x, y) \geq(\leq) F(x) G(y)$ for all $x, y \in \mathbf{R}$. The concepts

\footnotetext{
${ }^{1}$ Corresponding author: Department of Statistics, Ordered and Spatial Data Center of Excellence, Ferdowsi University of Mashhad, Mashhad, Iran. E-mail: Jabbarinh@um.ac.ir
}

Published 31 May 2016 / journal homepage: http://csam.or.kr

(C) 2016 The Korean Statistical Society, and Korean International Statistical Society. All rights reserved. 
of positive quadrant dependence (PQD) and negative quadrant dependence (NQD) are symmetric in $x$ and $y$.

Testing for independence between two continuous random variables $X$ and $Y$ is important when examining if two random variables are independent. As an natural case, we need to discover the dependence between two random characters to detect the interaction effects and finally controlling one of them. In this paper, we consider the problem of testing the null hypothesis of independence $H_{0}: H(x, y)=F(x) G(y)$ for all $x, y \in \mathbf{R}$ against the alternative hypothesis of strict PQD (NQD).

Rödel and Kössler (2004) compared the adaptive tests with some usual linear rank tests of independence such as Spearman rank correlation test, van der Waerden test, Fisher-Yates correlation test and exponential scores test and with the $t$-version of Pearson test. Genest and Verret (2005) provided a general formula for the locally most powerful rank test of independence against alternatives. Genest et al. (2006) considered the asymptotic behavior of the Cramér-von Mises test using the empirical copula process originally proposed by Deheuvels (1981).

Kochar and Gupta $(1987,1990)$ proposed a class of distribution-free tests for independence hypothesis against PQD. Shetty and Pandit (2003) introduced a class of distribution-free tests for independence. The proposed statistic by Kochar and Gupta (1990) is a member of this class. Güven and Kotz (2008) proposed a test of independence against PQD for a pair of absolutely continuous random variables jointly distributed according to the generalized FGM distribution. The result of Güven and Kotz (2008) is the asymptotic normality of their test statistic. The test maximizes the minimum power and possesses a monotone increasing power over the alternative hypothesis. Amini et al. $(2010,2011)$ used a dependence measure for the generalized FGM family in view of Kochar and Gupta (1987) and compared the power of tests proposed by them in absolutely continuous bivariate FGM distributions with those proposed by Güven and Kotz (2008).

The above authors have proposed a test of independence individually; however, these tests have not been compared. In this paper, we compare the tests in a generalized FGM family. For this purpose, we consider some linear rank tests, the locally most powerful rank test, the Cramér-von Mises test, the class of tests proposed by Kochar and Gupta (1987) and the test proposed by Güven and Kotz (2008) to test independence against strict PQD or NQD. We then estimate the power of the tests based on the asymptotic distribution of test statistics for each sample of size $n=20,30,50$ via simulation study.

Let $(X, Y)$ be a pair of absolutely continuous random variables with the marginals $F(x)$ and $G(y)$. According to Bairamov and Kotz (2002), the generalized FGM distribution function of $(X, Y)$ is

$$
H_{\theta}(x, y)=F(x) G(y)\left\{1+\theta\left(1-F^{p}(x)\right)^{q}\left(1-G^{p}(y)\right)^{q}\right\}, \quad \forall x, y \in \mathbf{R}, p \geq 1, q>1 .
$$

The admissible range of $\theta$ is

$$
-\min \left\{\frac{1}{p^{2}}\left(\frac{1+p q}{p(q-1)}\right)^{2(q-1)}, 1\right\} \leq \theta \leq \frac{1}{p}\left(\frac{1+p q}{p(q-1)}\right)^{q-1} .
$$

The copula function $C$ is a bivariate distribution function with uniform marginals on $[0,1]$, such that

$$
H(x, y)=C(F(x), G(y)) \quad \forall x, y \in \mathbf{R} .
$$

By Sklar's Theorem (1959), $C$ is unique if $F$ and $G$ are continuous. Thus, we can construct bivariate distributions $H(x, y)=C(F(x), G(y))$ with given marginals $F$ and $G$ by using copula $C$. The copula $C$ is given by

$$
C(x, y)=H\left(F^{-1}(x), G^{-1}(y)\right), \quad 0 \leq x, y \leq 1,
$$


where $F^{-1}$ and $G^{-1}$ are quasi-inverses of $F$ and $G$, respectively.

Using Sklar's Theorem (1959), we can write the distribution function $(1.1)$ with $[0,1]$ uniform marginals as

$$
C_{\theta}(x, y)=x y\left\{1+\theta\left(1-x^{p}\right)^{q}\left(1-y^{p}\right)^{q}\right\}, \quad 0 \leq x, y \leq 1, p \geq 1, q>1 .
$$

For $\theta>0(\theta<0), X$ and $Y$ are strictly PQD (NQD) and they are independent when $\theta=0$. In this article, we test $H_{0}: \theta=0$ against $H_{1}: \theta \geq \eta$ (or $H_{2}: \theta \leq \eta^{\prime}$ ) based on some test statistics, where $\eta>0$ and $\eta^{\prime}<0$ are located in interval (1.2). The independence hypothesis is considered under the copula of generalized FGM distributions. This approach is important for two reasons. First, it would provide a simple way to describe the family of generalized FGM distributions via the generalized FGM copulas. Second, it would emphasize on the fact that appropriate test statistics should be marginalfree, i.e. entirely non-parametric (typically based on the ranks of the observations). The type of marginal distributions are involved are rarely known.

The paper is organized as follows. In Section 2, we introduce some statistics and their asymptotic distributions. In Section 3, we compare the powers of the proposed tests graphically via simulation study and use the tests for numerical examples.

\section{The test statistics}

Let $\left(X_{i}, Y_{i}\right)$, for $i=1, \ldots, n$, be a random sample from the generalized FGM distribution function $H_{\theta}$ in (1.1) whose underlying copula function is $C_{\theta}$ in (1.3) and $\theta$ is dependence parameter. Rödel and Kössler (2004) gathered some of linear rank tests along with their asymptotic distributions. We use these four cases in this article as follows.

\subsection{The Spearman rank correlation test $\left(r_{s}\right)$}

The Pearson sample correlation is

$$
r_{p}=\frac{\sum_{i=1}^{n}\left(X_{i}-\bar{X}\right)\left(Y_{i}-\bar{Y}\right)}{\sqrt{\sum_{i=1}^{n}\left(X_{i}-\bar{X}\right)^{2}} \sqrt{\sum_{i=1}^{n}\left(Y_{i}-\bar{Y}\right)^{2}}},
$$

where $\bar{X}$ and $\bar{Y}$ are the sample means.

Let $\left(R_{i}, S_{i}\right)$ be the bivariate ranks corresponding to $\left(X_{i}, Y_{i}\right)$, for $i=1, \ldots, n$. If we replace $\left(R_{i}, S_{i}\right)$, for $i=1, \ldots, n$, in (2.1), then we get Spearman rank correlation:

$$
r_{s}=\frac{\sum_{i=1}^{n}\left(R_{i}-\bar{R}\right)\left(S_{i}-\bar{S}\right)}{\sqrt{\sum_{i=1}^{n}\left(R_{i}-\bar{R}\right)^{2}} \sqrt{\sum_{i=1}^{n}\left(S_{i}-\bar{S}\right)^{2}}}=1-\frac{6 \sum_{i=1}^{n}\left(R_{i}-S_{i}\right)^{2}}{n^{3}-n} .
$$

Under $H_{0}, r_{s}$ is $\mathrm{AN}(0,1 /(n-1))$, where AN denotes asymptotically normal (i.e. $\sqrt{n-1} r_{s} \stackrel{d}{\longrightarrow}$ $N(0,1)$ as $n \rightarrow \infty)$ (Kendall and Gibbons, 1990). The null hypothesis $H_{0}$ is rejected in favor of $H_{1}$ $\left(H_{2}\right)$ at the asymptotic level of significance $\alpha$ if $r_{s} \geq z_{1-\alpha}(n-1)^{-1 / 2}\left(r_{s} \leq-z_{1-\alpha}(n-1)^{-1 / 2}\right)$, where $z_{1-\alpha}$ is the $(1-\alpha)$-quantile of the standard normal distribution.

\subsection{The van der Waerden test $\left(r_{w}\right)$}

Replacing the data values $\left(X_{i}, Y_{i}\right)$ by $\left(\Phi^{-1}\left(R_{i} /(n+1)\right), \Phi^{-1}\left(S_{i} /(n+1)\right)\right)$, for $i=1, \ldots, n$, in (2.1), we get the van der Waerden $(\mathrm{VW})$ correlation $r_{w}$, where $\Phi(\cdot)$ is the standard normal distribution function. 
Under $H_{0}, r_{w}$ is $\mathrm{AN}(0,1 /(n-1))$ (Hájek and S̆idák, 1967). As before, $H_{0}$ is rejected in favor of $H_{1}$ $\left(H_{2}\right)$ at the asymptotic level of significance $\alpha$ if $r_{w} \geq z_{1-\alpha}(n-1)^{-1 / 2}\left(r_{w} \leq-z_{1-\alpha}(n-1)^{-1 / 2}\right)$.

\subsection{The Fisher-Yates correlation test $\left(r_{F Y}\right)$}

Substituting the data values $\left(X_{i}, Y_{i}\right)$ by $\left(V_{R_{i}}^{(n)}, V_{S_{i}}^{(n)}\right)$, for $i=1, \ldots, n$, in (2.1), we obtain the FisherYates correlation test $r_{F Y}$, where $V_{i}^{(n)}$ is the expectation of the $i$ th order statistic of a standard normal sample of size $n$. Under $H_{0}, r_{F Y}$ is $\mathrm{AN}(0,1 /(n-1))$ (Hájek and Šidák, 1967). As before, $H_{0}$ is rejected in favor of $H_{1}\left(H_{2}\right)$ at the asymptotic level of significance $\alpha$ if $r_{F Y} \geq z_{1-\alpha}(n-1)^{-1 / 2}\left(r_{F Y} \leq\right.$ $\left.-z_{1-\alpha}(n-1)^{-1 / 2}\right)$.

\subsection{The exponential scores test $\left(r_{e x}\right)$}

Replacing the data values $\left(X_{i}, Y_{i}\right)$ by $\left(E_{R_{i}}^{(n)}, E_{S_{i}}^{(n)}\right)$, for $i=1, \ldots, n$, in (2.1), we get the exponential scores correlation test $r_{e x}$, where $E_{i}^{(n)}$ is the expectation of the $i$ th order statistic of a standard exponential sample of size $n$. Under $H_{0}, r_{e x}$ is $\mathrm{AN}(0,1 /(n-1))$ (Hájek and S̆idák, 1967). $H_{0}$ is rejected in favour of $H_{1}\left(H_{2}\right)$ if $r_{e x} \geq z_{1-\alpha}(n-1)^{-1 / 2}\left(r_{e x} \leq-z_{1-\alpha}(n-1)^{-1 / 2}\right)$.

\subsection{The locally most powerful rank test (LMPR)}

Let $\left(X_{1}, Y_{1}\right), \ldots,\left(X_{n}, Y_{n}\right)$ be a random sample from the distribution function $H_{\theta}$ whose underlying copula function is $C_{\theta}$ in (1.3). The copula density function of $C_{\theta}(x, y)$ is

$$
c_{\theta}(x, y)=1+\theta w(x, y), \quad 0 \leq x, y \leq 1, p \geq 1, q>1,
$$

where $w(x, y)=r(x, p, q) r(y, p, q)$ and $r(x, p, q)=\left(1-x^{p}\right)^{q-1}\left(1-(1+p q) x^{p}\right)$.

Genest and Verret (2005) provided a statistic for the locally most powerful rank test of independence against alternatives expressed by copula models. They mentioned four conditions to obtain the locally most powerful rank test. At first, we check these conditions in generalized FGM family as:

1. The parameter space of $\theta$ in (1.2) is a closed interval and $\theta=0$ leads to the copula corresponding to independence.

2. If $\theta<\theta^{\prime}$, then $C_{\theta}(x, y) \leq C_{\theta^{\prime}}(x, y)$ for all $x, y \in(0,1)$.

3. For every $\theta$ in (1.2), $C_{\theta}$ is absolutely continuous and its copula density function $c_{\theta}$ is absolutely continuous as a function of $\theta$ for every $x, y \in(0,1)$.

4. $\dot{c}_{\theta}(x, y)=\partial c_{\theta}(x, y) / \partial \theta=w(x, y)$ is continuous in $\theta$ in a neighborhood of zero and

$$
\begin{aligned}
\lim _{\theta \rightarrow 0} \int_{(0,1)^{2}}\left|\dot{c}_{\theta}(x, y)\right| d x d y & =\int_{(0,1)^{2}}\left|\dot{c}_{0}(x, y)\right| d x d y \\
& =\int_{(0,1)^{2}}|w(x, y)| d x d y \leq\left(1+\frac{1+p q}{p+1}\right)^{2}<\infty .
\end{aligned}
$$

Since $\int_{0}^{1} \dot{c}_{0}(x, y) d x=\int_{0}^{1} \dot{c}_{0}(x, y) d y=0$ and $\int_{0}^{s} \int_{0}^{t} \dot{c}_{0}(x, y) d y d x=\int_{0}^{s} \int_{0}^{t} w(x, y) d y d x \geq 0$ for all $s, t \in(0,1)$, according to Genest and Verret (2005, Proposition 1), if $T(r, s)=E\left\{\dot{c}_{0}\left(B_{r}, B_{s}^{*}\right)\right\}$, where 
$B_{r}$ and $B_{s}^{*}$ are two independence random variables respectively distributed as $\operatorname{Beta}(r, n-r+1)$ and $\operatorname{Beta}(s, n-s+1)$ and

$$
T_{n}^{*}=\frac{1}{n} \sum_{i=1}^{n} T\left(R_{i}, S_{i}\right)
$$

where $\left(R_{1}, S_{1}\right), \ldots,\left(R_{n}, S_{n}\right)$ denote the ranks associated with the random sample $\left(X_{1}, Y_{1}\right), \ldots,\left(X_{n}, Y_{n}\right)$ from the distribution function $H_{\theta}$ in (1.1) whose underlying copula function is $C_{\theta}$ in (1.3), then under the null hypothesis, $\sqrt{n} T_{n}^{*}$ is $\mathrm{AN}\left(0, \sigma^{2}\right)$, where

$$
\sigma^{2}=\int_{(0,1)^{2}}\left\{\dot{c}_{0}(x, y)\right\}^{2} d x d y .
$$

In the following proposition, we obtain $T_{n}^{*}$ and $\sigma^{2}$ for generalized FGM distribution.

Proposition 1. Let $\left(R_{1}, S_{1}\right), \ldots,\left(R_{n}, S_{n}\right)$ denote the ranks associated with a random sample $\left(X_{1}, Y_{1}\right)$, $\ldots,\left(X_{n}, Y_{n}\right)$ from the distribution function $H_{\theta}$ in (1.1) whose underlying copula function is $C_{\theta}$ in (1.3) satisfying Conditions 1-4. Then

$$
T_{n}^{*}=\frac{1}{n} \sum_{i=1}^{n} T\left(R_{i}, S_{i}\right)=\frac{1}{n} \sum_{i=1}^{n} A\left(R_{i}\right) A\left(S_{i}\right),
$$

where

$$
A(a)=\sum_{k=0}^{q-1}\left(\begin{array}{c}
q-1 \\
k
\end{array}\right)(-1)^{k} \frac{\Gamma(n+1)}{\Gamma(a)}\left[\frac{\Gamma(p k+a)}{\Gamma(p k+n+1)}-(1+p q) \frac{\Gamma(p(k+1)+a)}{\Gamma(p(k+1)+n+1)}\right]
$$

and

$$
\sigma^{2}=\int_{(0,1)^{2}}\left\{\dot{c}_{0}(x, y)\right\}^{2} d x d y=\left[\operatorname{Beta}\left(\frac{1}{p}, 2 q-1\right) \frac{p q^{2}(1+p)}{(1+2 p q)(1+2 p q-p)}\right]^{2},
$$

where $\operatorname{Beta}(a, b)=\Gamma(a) \Gamma(b) / \Gamma(a+b)$.

Proof: Since $\dot{c}_{\theta}(x, y)=w(x, y)$ and $B_{r} \sim \operatorname{Beta}(r, n-r+1)$ and $B_{s}^{*} \sim \operatorname{Beta}(s, n-s+1)$ are independent, we have

$$
\begin{aligned}
T(r, s) & =E\left\{\dot{c}_{0}\left(B_{r}, B_{s}^{*}\right)\right\}=E\left\{w\left(B_{r}, B_{s}^{*}\right)\right\} \\
& =E\left\{r\left(B_{r}, p, q\right) r\left(B_{s}^{*}, p, q\right)\right\}=E\left\{r\left(B_{r}, p, q\right)\right\} E\left\{r\left(B_{s}^{*}, p, q\right)\right\} .
\end{aligned}
$$

Now,

$$
\begin{aligned}
E\left\{r\left(B_{r}, p, q\right)\right\} & =E\left\{\left(1-B_{r}^{p}\right)^{q-1}\left(1-(1+p q) B_{r}^{p}\right)\right\} \\
& =E\left\{\left(1-B_{r}^{p}\right)^{q-1}\right\}-(1+p q) E\left\{\left(1-B_{r}^{p}\right)^{q-1} B_{r}^{p}\right\} .
\end{aligned}
$$

Using the binomial expansion $(1-a)^{n}=\sum_{k=0}^{n}\left(\begin{array}{l}n \\ k\end{array}\right)(-1)^{k} a^{k}$, we can compute $E\left\{r\left(B_{r}, p, q\right)\right\}, E\left\{r\left(B_{s}^{*}, p, q\right)\right\}$ and $T(r, s)$. So, we can obtain $T_{n}^{*}$. 
For computing $\sigma^{2}$, we have

$$
\begin{aligned}
\sigma^{2} & =\int_{(0,1)^{2}}\left\{\dot{c}_{0}(x, y)\right\}^{2} d x d y=\int_{(0,1)^{2}} w^{2}(x, y) d x d y \\
& =\int_{(0,1)^{2}} r^{2}(x, p, q) r^{2}(y, p, q) d x d y=\left[\int_{0}^{1}\left(1-x^{p}\right)^{2(q-1)}\left(1-(1+p q) x^{p}\right)^{2} d x\right]^{2} .
\end{aligned}
$$

We can obtain $\sigma^{2}$ by a straightforward computation if we make the substitution $u=x^{p}$. This completes the proof.

According to Genest and Verret (2005), $H_{0}$ is rejected against $H_{1}\left(H_{2}\right)$ for large (small) enough values of $T_{n}^{*}$. The rejection region of $H_{0}$ in favor of $H_{1}\left(H_{2}\right)$ is $T_{n}^{*} \geq z_{1-\alpha}(\sigma / \sqrt{n})\left(T_{n} \leq-z_{1-\alpha}(\sigma / \sqrt{n})\right)$, where $z_{1-\alpha}$ is the $(1-\alpha)$-quantile of the standard normal distribution.

\subsection{The Cramér-von Mises test (CvM)}

Let $\left(X_{1}, Y_{1}\right), \ldots,\left(X_{n}, Y_{n}\right)$ be a random sample from the distribution function $H_{\theta}$ in (1.1) whose underlying copula function is $C_{\theta}$ in $(1.3)$ and $\left(R_{1}, S_{1}\right), \ldots,\left(R_{n}, S_{n}\right)$ denote the ranks associated with this random sample. Genest et al. (2006) represented a powerful nonparametric test of independence based on the Cramér-von Mises statistic

$$
B_{n}=\frac{1}{n} \sum_{i=1}^{n} \sum_{j=1}^{n} D_{n}\left(R_{i}, R_{j}\right) D_{n}\left(S_{i}, S_{j}\right),
$$

where

$$
D_{n}(s, t)=\frac{2 n+1}{6 n}+\frac{s(s-1)}{2 n(n+1)}+\frac{t(t-1)}{2 n(n+1)}-\frac{\max (s, t)}{n+1} .
$$

The null hypothesis $H_{0}$ is rejected in favor of $H_{1}$ for large enough values of $B_{n}$. So, the critical values of the test at level $\alpha$ is obtained using $P_{\theta=0}\left(B_{n}>c\right)=\alpha$. According to Genest and Rémillard (2004), for finite $n$, we compute the empirical critical values $c$ using 20000 independent replications under the null hypothesis of independence or randomness. The empirical critical values $c$ at level $\alpha=0.05$ based on a random sample of sizes $n=20,30,50$ are $0.0527,0.0545,0.0559$, respectively.

Now, if we assume $(X, Y)$ is NQD, then $(X,-Y)$ is PQD (Nelsen, 2006). Thus, for testing independence hypothesis $H_{0}: \theta=0$ against $\operatorname{NQD}\left(H_{2}: \theta \leq \eta^{\prime}\right)$, it suffices to test $H_{0}: \theta=0$ against $H_{1}: \theta \geq-\eta^{\prime}$ using $B_{n}$ in (2.4) for observations $\left(X_{i},-Y_{i}\right), i=1,2, \ldots, n$.

\subsection{The Kochar and Gupta class of distribution-free tests}

For testing independence against PQD (NQD), Kochar and Gupta (1987) introduced $\delta_{k}(x, y)=H^{k}(x, y)$ $F^{k}(x) G^{k}(y)$ for all $x, y \in \mathbf{R}$, where $k$ is a fixed integer. Under $H_{0}, \delta_{k}(x, y)=0$ and under $H_{1}\left(H_{2}\right)$, $\delta_{k}(x, y) \geq 0\left(\delta_{k}(x, y) \leq 0\right)$ for all $x, y \in \mathbf{R}$ and with a strict inequality over a set of nonzero probability. The measure of deviation between $H_{0}$ and $H_{1}\left(H_{2}\right)$ is then

$$
D_{k}=\int_{R^{2}} \delta_{k}(x, y) d H(x, y)=D_{1 k}-D_{2 k},
$$

where $D_{1 k}=\int_{R^{2}} H^{k}(x, y) d H(x, y)=P\left(\max \left(X_{1}, \ldots, X_{k}\right) \leq X_{k+1}, \max \left(Y_{1}, \ldots, Y_{k}\right) \leq Y_{k+1}\right), D_{2 k}=\int_{R^{2}}$ $F^{k}(x) G^{k}(y) d H(x, y)=\int_{R^{2}} \bar{H}(x, y) d F^{k}(x) d G^{k}(y)$ and $\bar{H}(x, y)=P(X>x, Y>y)$. 
Amini et al. (2011) showed that $D_{1 k}$ and $D_{2 k}$ are

$$
\begin{aligned}
D_{1 k}= & \sum_{j=0}^{k}\left(\begin{array}{l}
k \\
j
\end{array}\right) \frac{\theta^{j}}{p^{2}} \operatorname{Beta}^{2}\left(\frac{k+1}{p}, j q+1\right) \\
& +\sum_{j=0}^{k}\left(\begin{array}{l}
k \\
j
\end{array}\right) \frac{\theta^{j+1}}{p^{2}}\left[\operatorname{Beta}\left(\frac{k+1}{p},(j+1) q\right)-(1+p q) \operatorname{Beta}\left(\frac{k+1}{p}+1,(j+1) q\right)\right]^{2}
\end{aligned}
$$

and

$$
D_{2 k}=\frac{1}{(k+1)^{2}}+\frac{\theta}{p^{2}}\left[\operatorname{Beta}\left(\frac{k+1}{p}, q\right)-(1+p q) \operatorname{Beta}\left(\frac{k+1}{p}+1, q\right)\right]^{2}
$$

in generalized FGM family where, $\operatorname{Beta}(a, b)=\Gamma(a) \Gamma(b) / \Gamma(a+b)$

Under $H_{0}, D_{1 k}=D_{2 k}=(k+1)^{-2}$ but, under $H_{1}\left(H_{2}\right), D_{1 k}>D_{2 k}>(k+1)^{-2}\left(D_{1 k}<D_{2 k}<(k+1)^{-2}\right)$. Let $\left(X_{i}, Y_{i}\right)$, for $i=1, \ldots, n$, be a random sample of generalized FGM distribution and suppose that

$$
\varphi_{k+1}\left\{\left(X_{1}, Y_{1}\right), \ldots,\left(X_{k+1}, Y_{k+1}\right)\right\}= \begin{cases}1, & \left\{\max \left(X_{1}, \ldots, X_{k+1}\right), \max \left(Y_{1}, \ldots, Y_{k+1}\right)\right\} \\ & \text { belongs to the same pair of }(X, Y), \\ 0, & \text { otherwise. }\end{cases}
$$

For testing $H_{0}$ against $H_{1}\left(H_{2}\right)$, we use U-statistic estimator of $D_{1 k}$, that is

$$
U_{n}(k+1)=\frac{1}{\left(\begin{array}{c}
n \\
k+1
\end{array}\right)} \sum \varphi_{k+1}\left\{\left(X_{i_{1}}, Y_{i_{1}}\right), \ldots,\left(X_{i_{k+1}}, Y_{i_{k+1}}\right)\right\},
$$

where the sum is over all combinations of $(k+1)$ integers $\left(i_{1}, \ldots, i_{k+1}\right)$ chosen out of $(1, \ldots, n)$.

Notice that the large (small) values of $U_{n}(k+1)$ are significant for testing $H_{0}$ against $H_{1}\left(H_{2}\right)$. For $k=1$, we also have $U_{n}(2)=\left(\tau_{n}+1\right) / 2$, where $\tau_{n}$ is Kendall's statistic. So, the independence test based on $U_{n}(2)$ is equivalent to the classical test based on $\tau_{n}$.

The asymptotic distribution of $\sqrt{n}\left\{U_{n}(k+1)-E\left(U_{n}(k+1)\right)\right\}$ is normal with expectation zero and variance $\sigma_{k+1}^{2}=(k+1)^{2} \gamma_{1}$, where

$$
\gamma_{1}=E\left\{\psi_{1}^{2}\left(X_{1}, Y_{1}\right)\right\}-E^{2}\left\{U_{n}(k+1)\right\}, \quad \psi_{1}\left(x_{1}, y_{1}\right)=E\left[\varphi_{k+1}\left\{\left(x_{1}, y_{1}\right), \ldots,\left(X_{k+1}, Y_{k+1}\right)\right\}\right],
$$

see Serfling (1980).

Under $H_{0}, E\left(U_{n}(k+1)\right)=(k+1)^{-1}, \gamma_{1}=k^{2} /\left\{(2 k+1)^{2}(k+1)^{2}\right\}$ and $\sigma_{k+1}^{2}=k^{2}(2 k+1)^{-2} . H_{0}$ is rejected in favor of $H_{1}$ if $U_{n}(k+1)>z_{1-\alpha}[\sqrt{n}(2 k+1)]^{-1} k+(k+1)^{-1}$.

Now, if we assume $(X, Y)$ is NQD, then $(X,-Y)$ is PQD (Nelsen, 2006). Thus, for testing independence hypothesis $H_{0}: \theta=0$ against strict $\operatorname{NQD}\left(H_{2}: \theta \leq \eta^{\prime}\right)$, it suffices to test the hypothesis $H_{0}: \theta=0$ against $H_{1}: \theta \geq-\eta^{\prime}$ using $\varphi_{k+1}$ in (2.7) for observations $\left(X_{i},-Y_{i}\right), i=1,2, \ldots, n$. To obtain the exact distribution of $U_{n}(k+1)$, see Kochar and Gupta (1987).

\subsection{The Güven and Kotz test}

Let $\left(X_{1}, Y_{1}\right), \ldots,\left(X_{n}, Y_{n}\right)$ be a random sample from the distribution function $H_{\theta}$ whose underlying copula function is $C_{\theta}$ in (1.3) and the copula density function $c_{\theta}$ in (2.3). Güven and Kotz (2008) proposed the following statistic for testing independence $\left(H_{0}: \theta=0\right)$ against $\operatorname{PQD}\left(H_{1}: \theta \geq \eta\right)$ as

$$
\phi(\mathbf{x}, \mathbf{y})= \begin{cases}1, & T \geq c, \\ 0, & T<c,\end{cases}
$$


where $\eta \in\left(0,(1 / p)\{(1+p q) / p(q-1)\}^{q-1}\right], T=\prod_{i=1}^{n}\left(1+\eta w\left(X_{i}, Y_{i}\right)\right)$.

The asymptotic distribution of $T$ is

$$
\sqrt{n}\left(T^{\frac{1}{n}}-\mu(\theta)\right) \stackrel{d}{\longrightarrow} N\left(0, \sigma^{2}(\theta)\right) \text { as } n \rightarrow \infty,
$$

where $\mu(\theta)=\exp [E(\ln (1+\eta w(X, Y)))]$ and $\sigma^{2}(\theta)=\mu^{2}(\theta) \operatorname{Var}[\ln (1+\eta w(X, Y))]$.

The rejection region of $H_{0}$ in favor of $H_{1}$ is $T \geq c_{\alpha}$, where $c_{\alpha}^{1 / n}=z_{1-\alpha} \sigma(0) / \sqrt{n}+\mu(0)$. An approximation to the power of the test for finite sample size is given by

$$
\beta_{n}(\theta) \simeq 1-\Phi\left(\frac{\sqrt{n}}{\sigma(\theta)}\left[c_{\alpha}^{\frac{1}{n}}-\mu(\theta)\right]\right)
$$

where $\theta \in\left[\eta,(1 / p)\{(1+p q) / p(q-1)\}^{q-1}\right]$ and $\Phi(\cdot)$ is the standard normal distribution function.

Now, if we assume $(X, Y)$ is NQD, then $(X,-Y)$ is PQD (Nelsen, 2006). Thus, for testing independence $\left(H_{0}: \theta=0\right)$ against $\operatorname{NQD}\left(H_{2}: \theta \leq \eta^{\prime}\right)$ where $\eta^{\prime} \in\left[-\min \left\{\left(1 / p^{2}\right)((1+p q) / p(q-1))^{2(q-1)}\right.\right.$, $1\}, 0)$, it suffices to test the hypothesis $H_{0}: \theta=0$ against $H_{1}: \theta \geq-\eta^{\prime}$ using $\phi(\mathbf{x}, \mathbf{y})$ in (2.8) for observations $\left(X_{i},-Y_{i}\right), i=1,2, \ldots, n$.

\section{Simulation results and applied examples}

In this section, we compare the power of tests introduced in Section 2 to test the independence hypothesis against PQD (NQD). There is a wide applications of generalized FGM distribution for values of $p=2$ and $q=2$; consequently, some of authors such as Jung et al. (2008) and Güven and Kotz (2008) consider this case of the generalized FGM family. In addition, we have calculated the power of test statistics for other values of $p$ and $q$ such as " $p=2$ and $q=3$ " and " $p=3$ and $q=2$ ". The results for these values of $p$ and $q$ have a similar process. So for the sake of simplicity, we consider the information related to $p=q=2$ case. We evaluate the empirical powers of test statistics $r_{s}, r_{w}$, $r_{F Y}, r_{e x}, T_{n}^{*}(\mathrm{LMPR}), B_{n}(\mathrm{CvM}), U_{n}(k+1)$ for $k=1,2,3,4$ and $T$ based on the simulation results for 5000 replications using R software version 3.0.1 at 5\% level of significance. Notice that, for $n=20$, the critical points are calculated based on exact distribution of $U_{n}(k+1)$ (Kochar and Gupta's statistic) and for $n=30,50$, the critical points are calculated based on approximate distribution of $U_{n}(k+1)$. Also, the critical points of $T$ (Güven and Kotz's statistic) based on asymptotic distribution with various values of $\eta \in(0,1.25], \theta \in[\eta, 1.25]$ for PQD case and $\eta^{\prime} \in[-1,0), \theta \in\left[-1, \eta^{\prime}\right]$ for NQD case is obtained (notice that according to (1.2) for $p=q=2$, the range of $\theta$ is $[-1,1.25]$ ). Table 1 shows the empirical powers for $p=q=2, \eta=0.2$ and $\eta^{\prime}=-0.2$. Notice that the empirical sizes are closed to 0.05 for all test statistics. This point is important for choosing the best test statistic.

\subsection{Results in PQD case}

A) Comparing Kochar and Gupta's statistics:

When $n \geq 20, U_{n}(2)$ is the best test statistic, except for $n=30$ and $\theta=0.2$, for which $U_{n}(3)$ is the best test statistic but its power is very close to $U_{n}(2)$. Also, we have the following order $U_{n}(5)<U_{n}(4)<U_{n}(3)<U_{n}(2)$ based on the powers of these test statistics (Table 1).

B) Comparing the rank tests (Table 1):

- When $n=20$ for all $\theta, r_{s}$ is better than all of rank tests, except for $\theta=1.2$, for which LMPR test is the best one. 
Table 1: Empirical powers for marginals $U(0,1)$ in the generalized FGM family for NQD and PQD cases $\left(p=q=2, \eta=0.2\right.$ and $\left.\eta^{\prime}=-0.2\right)$

\begin{tabular}{|c|c|c|c|c|c|c|c|c|c|c|c|}
\hline \multirow{2}{*}{$n$} & \multirow{2}{*}{ Statistics } & \multicolumn{10}{|c|}{$\theta$} \\
\hline & & -1 & -0.7 & -0.5 & -0.2 & 0 & 0.2 & 0.5 & 0.7 & 1 & 1.2 \\
\hline \multirow{11}{*}{20} & $r_{s}$ & 0.402 & 0.255 & 0.165 & 0.081 & 0.051 & 0.096 & 0.167 & 0.258 & 0.415 & 0.538 \\
\hline & $r_{w}$ & 0.351 & 0.231 & 0.144 & 0.078 & 0.049 & 0.090 & 0.161 & 0.236 & 0.374 & 0.485 \\
\hline & $r_{F Y}$ & 0.338 & 0.223 & 0.141 & 0.075 & 0.050 & 0.089 & 0.157 & 0.231 & 0.363 & 0.467 \\
\hline & $r_{e x}$ & 0.197 & 0.132 & 0.084 & 0.043 & 0.070 & 0.090 & 0.115 & 0.156 & 0.197 & 0.237 \\
\hline & LMPR & 0.402 & 0.227 & 0.140 & 0.057 & 0.026 & 0.063 & 0.136 & 0.213 & 0.401 & 0.560 \\
\hline & CvM & 0.316 & 0.177 & 0.110 & 0.056 & 0.052 & 0.056 & 0.110 & 0.167 & 0.328 & 0.455 \\
\hline & $T$ & 0.585 & 0.353 & 0.226 & 0.100 & 0.045 & 0.118 & 0.236 & 0.363 & 0.589 & 0.728 \\
\hline & $U_{n}(2)$ & 0.381 & 0.245 & 0.155 & 0.080 & 0.050 & 0.091 & 0.167 & 0.250 & 0.397 & 0.513 \\
\hline & $U_{n}(3)$ & 0.276 & 0.188 & 0.125 & 0.072 & 0.050 & 0.082 & 0.138 & 0.197 & 0.297 & 0.371 \\
\hline & $U_{n}(4)$ & 0.224 & 0.158 & 0.111 & 0.067 & 0.048 & 0.075 & 0.110 & 0.147 & 0.202 & 0.241 \\
\hline & $U_{n}(5)$ & 0.192 & 0.141 & 0.098 & 0.063 & 0.046 & 0.068 & 0.089 & 0.115 & 0.147 & 0.164 \\
\hline \multirow{11}{*}{30} & $r_{s}$ & 0.554 & 0.331 & 0.216 & 0.097 & 0.047 & 0.094 & 0.228 & 0.332 & 0.550 & 0.712 \\
\hline & $r_{w}$ & 0.482 & 0.289 & 0.183 & 0.090 & 0.047 & 0.090 & 0.205 & 0.294 & 0.487 & 0.637 \\
\hline & $r_{F Y}$ & 0.469 & 0.284 & 0.178 & 0.088 & 0.065 & 0.090 & 0.200 & 0.287 & 0.473 & 0.620 \\
\hline & $r_{e x}$ & 0.248 & 0.159 & 0.103 & 0.053 & 0.047 & 0.087 & 0.127 & 0.153 & 0.235 & 0.288 \\
\hline & LMPR & 0.630 & 0.366 & 0.213 & 0.077 & 0.034 & 0.081 & 0.216 & 0.361 & 0.622 & 0.792 \\
\hline & CvM & 0.482 & 0.260 & 0.148 & 0.062 & 0.049 & 0.075 & 0.166 & 0.267 & 0.504 & 0.679 \\
\hline & $T$ & 0.751 & 0.466 & 0.291 & 0.109 & 0.049 & 0.106 & 0.295 & 0.473 & 0.736 & 0.887 \\
\hline & $U_{n}(2)$ & 0.527 & 0.307 & 0.202 & 0.088 & 0.046 & 0.093 & 0.224 & 0.330 & 0.543 & 0.697 \\
\hline & $U_{n}(3)$ & 0.392 & 0.249 & 0.169 & 0.091 & 0.065 & 0.105 & 0.207 & 0.285 & 0.451 & 0.567 \\
\hline & $U_{n}(4)$ & 0.273 & 0.182 & 0.125 & 0.070 & 0.073 & 0.090 & 0.166 & 0.218 & 0.334 & 0.410 \\
\hline & $U_{n}(5)$ & 0.193 & 0.136 & 0.095 & 0.056 & 0.082 & 0.086 & 0.148 & 0.178 & 0.254 & 0.307 \\
\hline \multirow{11}{*}{50} & $r_{s}$ & 0.767 & 0.501 & 0.310 & 0.115 & 0.050 & 0.103 & 0.307 & 0.492 & 0.758 & 0.892 \\
\hline & $r_{w}$ & 0.675 & 0.426 & 0.267 & 0.100 & 0.051 & 0.096 & 0.266 & 0.427 & 0.673 & 0.818 \\
\hline & $r_{F Y}$ & 0.660 & 0.414 & 0.260 & 0.097 & 0.053 & 0.097 & 0.256 & 0.417 & 0.655 & 0.806 \\
\hline & $r_{e x}$ & 0.333 & 0.216 & 0.138 & 0.060 & 0.060 & 0.079 & 0.143 & 0.199 & 0.298 & 0.377 \\
\hline & LMPR & 0.877 & 0.580 & 0.346 & 0.115 & 0.044 & 0.116 & 0.361 & 0.587 & 0.863 & 0.962 \\
\hline & CvM & 0.730 & 0.421 & 0.229 & 0.079 & 0.053 & 0.073 & 0.235 & 0.413 & 0.730 & 0.887 \\
\hline & $T$ & 0.923 & 0.662 & 0.424 & 0.152 & 0.054 & 0.140 & 0.420 & 0.658 & 0.915 & 0.980 \\
\hline & $U_{n}(2)$ & 0.756 & 0.494 & 0.307 & 0.115 & 0.055 & 0.109 & 0.318 & 0.509 & 0.764 & 0.895 \\
\hline & $U_{n}(3)$ & 0.553 & 0.359 & 0.239 & 0.096 & 0.065 & 0.105 & 0.255 & 0.392 & 0.605 & 0.735 \\
\hline & $U_{n}(4)$ & 0.366 & 0.245 & 0.165 & 0.077 & 0.072 & 0.094 & 0.200 & 0.288 & 0.434 & 0.550 \\
\hline & $U_{n}(5)$ & 0.250 & 0.178 & 0.127 & 0.020 & 0.074 & 0.088 & 0.162 & 0.222 & 0.319 & 0.394 \\
\hline
\end{tabular}

FGM = Farlie-Gumbel-Morgenstern, NQD = negative quadrant dependence, PQD = positive quadrant dependence, LMPR = locally most powerful rank test, CvM = Cramér-von Mises test.

- When $n=30$ for $\theta=0.2,0.5, r_{s}$ is the best competitor and for $\theta=0.7,1,1.2$, LMPR test is the best one.

- When $n=50$ (large samples) for all $\theta$, LMPR test is better than other rank tests.

- For all $\theta$ and $n$, LMPR test is better than the CvM test.

- For all $n$ and $\theta, r_{w}$ and $r_{F Y}$ are better than $r_{e x}$.

C) Comparison of all statistics:

Figures 1-3 show the power of all statistics (rank tests, $T$ and $U_{n}(k+1)$ for $k=1,2,3,4$ ) for some values of $\eta$ with respect to $\theta$ when $n=20,30,50$ (notice that we don't show powers of $U_{n}(3)$, $U_{n}(4)$ and $U_{n}(5)$ in figures because their powers are less than $\left.U_{n}(2)\right)$. The results are

- $\theta$ and $\eta, T$ is the best test statistic for all $n$. 


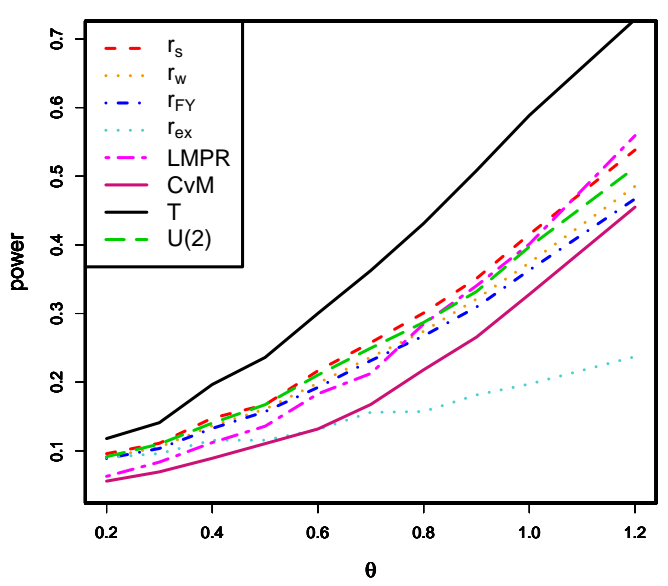

(a) $n=20$ and $\eta=0.2$

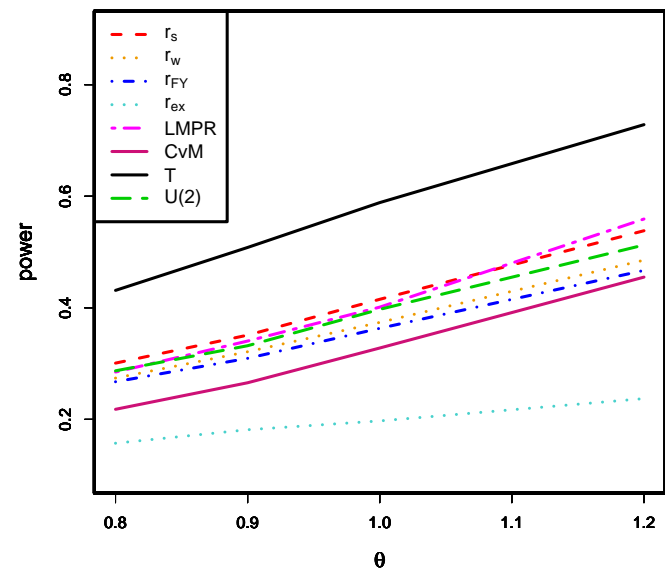

(b) $n=20$ and $\eta=0.8$

Figure 1: The empirical power of all tests with respect to $\theta$ for $n=20$ and $\eta=0.2,0.8$.

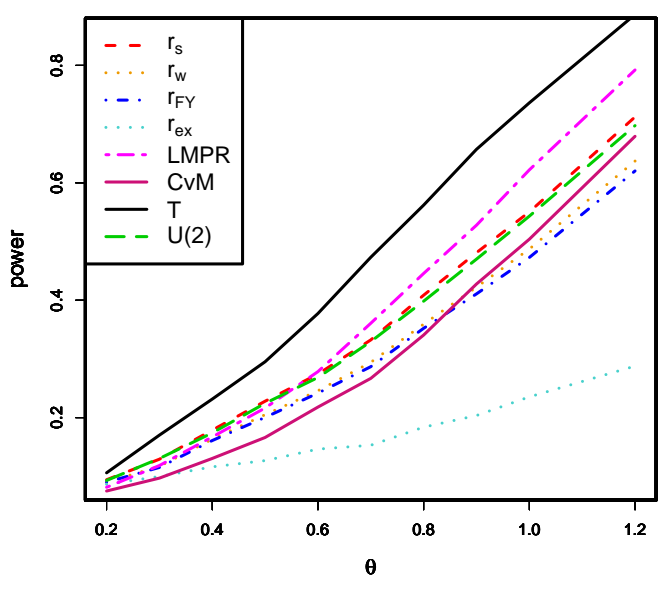

(a) $n=30$ and $\eta=0.2$

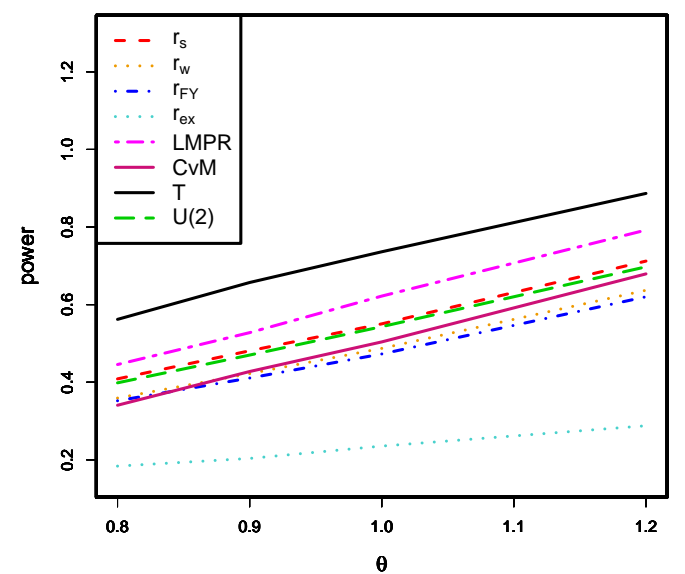

(b) $n=30$ and $\eta=0.8$

Figure 2: The empirical power of all tests with respect to $\theta$ for $n=30$ and $\eta=0.2,0.8$.

- For all $n$, the powers of $r_{s}$ and $U_{n}(2)$ are close to $T$ when $\theta$ is small (weak dependence).

- For all $n, T$ is a appropriate test statistic when $\theta$ is large (strong dependence).

- The powers of $T$ statistic and LMPR test are greater than other tests when $n=50$ (large samples); in addition, the power of LMPR is close to $T$ if $\theta$ is small or large.

- The power of $U_{n}(2)$ is very close to the power of $r_{s}$ when $n=50$.

\subsection{Results in NQD case}

A) Comparing Kochar and Gupta's statistics: 


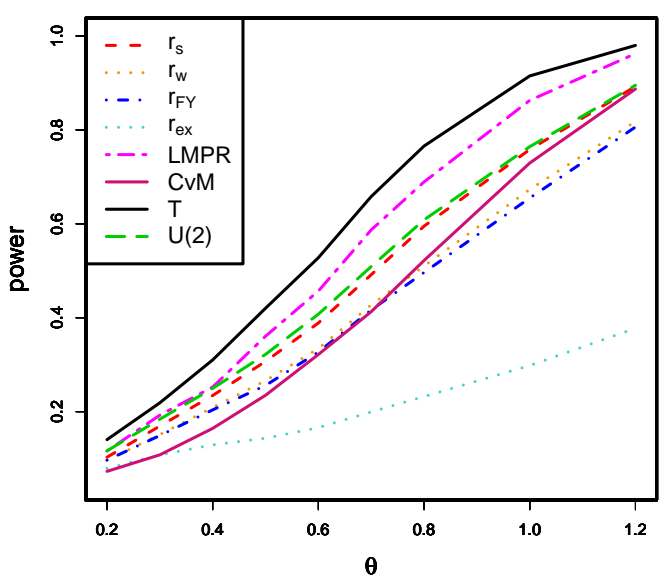

(a) $n=50$ and $\eta=0.2$

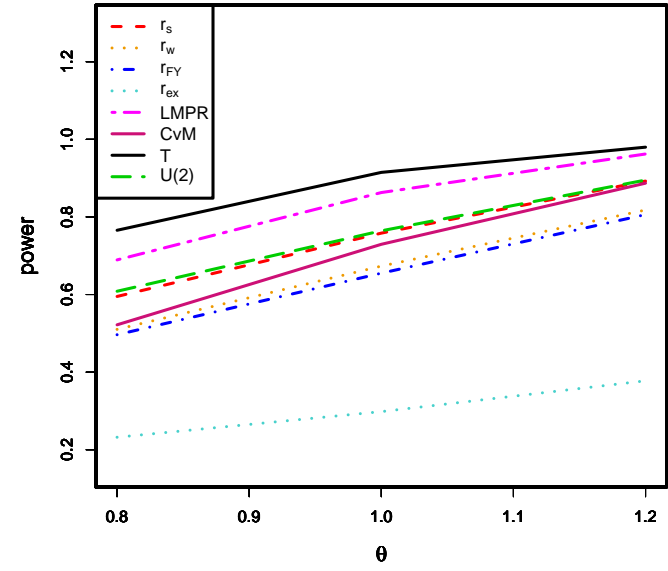

(b) $n=50$ and $\eta=0.8$

Figure 3: The empirical power of all tests with respect to $\theta$ for $n=50$ and $\eta=0.2,0.8$.

When $n \geq 20, U_{n}(2)$ is the best test statistic, except for $n=30$ and $\theta=-0.2$, for which $U_{n}(3)$ is the best test statistic but its power is very close to $U_{n}(2)$. Also, for all $n$, we have the following order $U_{n}(5)<U_{n}(4)<U_{n}(3)<U_{n}(2)$ based on the powers of these test statistics (see Table 1).

B) Comparing the rank tests (Table 1):

- $r_{s}$ is better than all of rank tests when $n=20$ for all $\theta$.

- $r_{s}$ is the best competitor and for $\theta=-0.7,-1$, LMPR test is the best one when $n=30$ for $\theta=-0.2,-0.5$.

- LMPR test is better than other rank tests when $n=50$ (large samples) for all $\theta$.

- LMPR test is better than CvM test for all $\theta$ and $n$.

- $r_{w}$ and $r_{F Y}$ are better than $r_{e x}$ for all $n$ and $\theta$.

C) Comparison of all statistics:

Figures 4-6 show the power of all statistics (rank tests, $T$ and $U_{n}(k+1)$ for $k=1,2,3,4$ ) for some values of $\eta^{\prime}$ with respect to $\theta$ when $n=20,30,50$ (notice that we do not show the power of $U_{n}(3)$, $U_{n}(4)$ and $U_{n}(5)$ in figures because their powers are less than $\left.U_{n}(2)\right)$. The results are

- $\theta$ and $\eta^{\prime}, T$ is the best test statistic for all $n$.

- The powers of $r_{s}$ and $U_{n}(2)$ are close to $T$ for all $n$, when $|\theta|$ is small (weak dependence).

- $T$ is an appropriate test statistic for all $n$ when $|\theta|$ is large (strong dependence).

- The powers of $T$ statistic and LMPR test are greater than other tests when $n=50$ (large samples); in addition, the power of LMPR is close to $T$ if $\theta$ is small or large.

- The power of $U_{n}(2)$ is very close to the power of $r_{s}$ when $n=50$. 


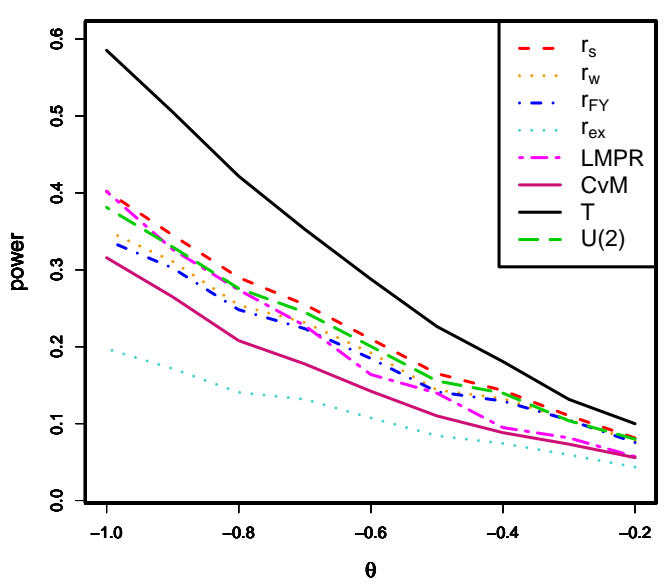

(a) $n=20$ and $\eta^{\prime}=-0.2$

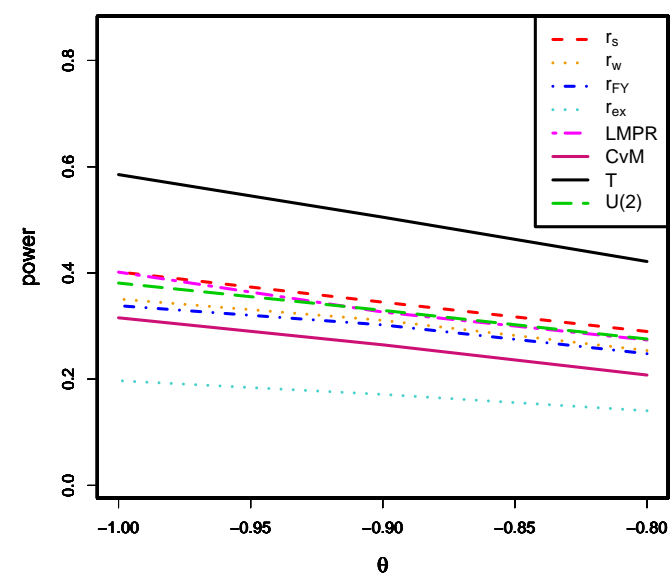

(b) $n=20$ and $\eta^{\prime}=-0.8$

Figure 4: The empirical power of all tests with respect to $\theta$ for $n=20$ and $\eta^{\prime}=-0.2,-0.8$.

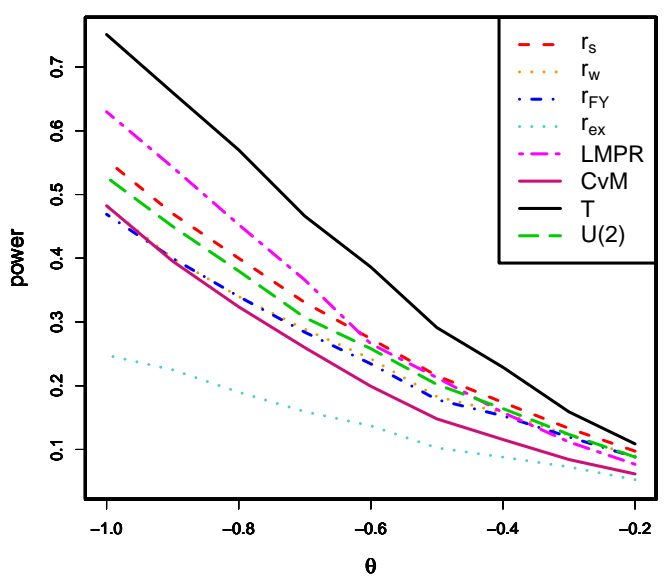

(a) $n=30$ and $\eta^{\prime}=-0.2$

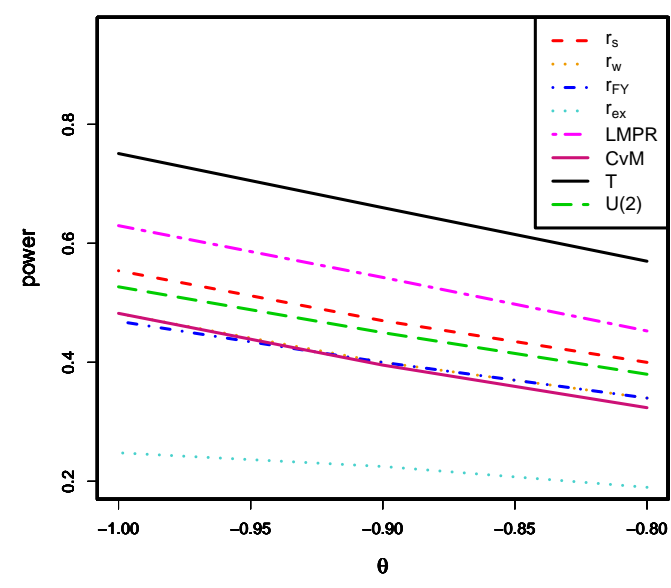

(b) $n=30$ and $\eta^{\prime}=-0.8$

Figure 5: The empirical power of all tests with respect to $\theta$ for $n=30$ and $\eta^{\prime}=-0.2,-0.8$.

\subsection{Examples}

Cook and Johnson (1986) considered the analysis of the uranium exploration data set. These data consist of 655 chemical analyses from water samples collected from the Montrose quadrangle of western Colorado. Concentrations were measured for the following elements: uranium (U), lithium (Li), cobalt (Co), potassium (K), cesium (Cs), scandium (Sc) and titanium (Ti).

Peacock (1983) and Fasano and Franceschini (1987) proposed a generalization of the classical Kolmogorov-Smirnov test which is suitable to analyze random samples defined in two dimensions. In their method for any data point of $\left(X_{i}, Y_{i}\right)$, we consider the four quadrants of the plane defined by

$$
\left(x<X_{i}, y<Y_{i}\right), \quad\left(x<X_{i}, y>Y_{i}\right), \quad\left(x>X_{i}, y<Y_{i}\right), \quad\left(x>X_{i}, y>Y_{i}\right) \quad i=1, \ldots, n .
$$




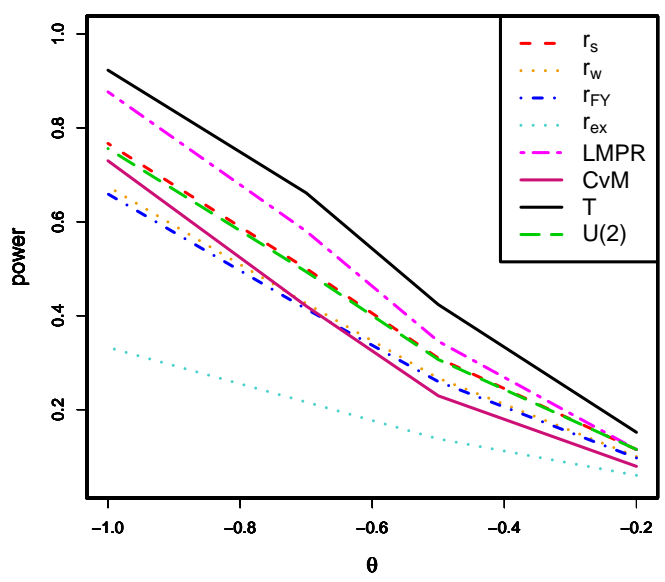

(a) $n=50$ and $\eta^{\prime}=-0.2$

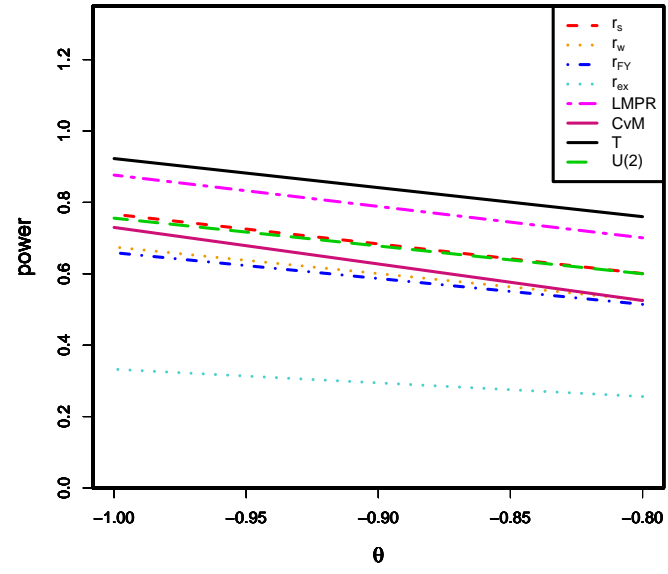

(b) $n=50$ and $\eta^{\prime}=-0.8$

Figure 6: The empirical power of all tests with respect to $\theta$ for $n=50$ and $\eta^{\prime}=-0.2,-0.8$.

Table 2: Goodness-of-fit test for two selected pairs of variables in uranium exploration data

\begin{tabular}{ccccccc}
\hline \hline Pair & $p$ & $q$ & $\operatorname{MLE}(\theta)$ & $-\ell$ & $D_{k s}$ & $p$-value \\
\hline$\left(U, C_{s}\right)$ & 2 & 2 & 0.464 & -0.9962 & 0.8203 & 0.827 \\
$(U, L i)$ & 2 & 2 & -0.430 & -0.7495 & 1.0496 & 0.481 \\
\hline \hline
\end{tabular}

MLE = maximum likelihood estimator.

Table 3: Values of test statistics, their critical values and associated $p$-values for selected pair $\left(U, C_{s}\right)$ in PQD case

\begin{tabular}{c|ccccccccccc}
\hline \hline & $r_{s}$ & $r_{w}$ & $r_{F y}$ & $r_{e x}$ & LMPR & CvM & $T$ & $U_{n}(2)$ & $U_{n}(3)$ & $U_{n}(4)$ & $U_{n}(5)$ \\
\hline Test statistic & 0.317 & 0.338 & 0.337 & 0.320 & 0.085 & 0.055 & 2.690 & 0.608 & 0.469 & 0.401 & 0.360 \\
Critical point & 0.234 & 0.234 & 0.234 & 0.234 & 0.094 & 0.056 & 3.713 & 0.577 & 0.426 & 0.349 & 0.303 \\
$p$-value & 0.013 & 0.008 & 0.009 & 0.012 & 0.068 & 0.052 & 0.078 & 0.010 & 0.008 & 0.006 & 0.005 \\
\hline \hline
\end{tabular}

$\mathrm{PQD}$ = positive quadrant dependence, LMPR = locally most powerful rank test, CvM = Cramér-von Mises test.

Then, we calculate the maximum absolute difference between the observed and predicted cumulative distributions (both normalized to 1) within all four quadrants. The Kolmogorov-Smirnov test statistic $D_{k s}$ is the maximum of these differences when all data points are considered.

We use the first 50 cases from two selected pairs $\left(U, C_{s}\right)$ and $(U, L i)$ in the uranium exploration data. First, we test whether one can fit generalized FGM distribution to $\left(U, C_{s}\right)$ and $(U, L i)$. For generalized FGM with $p=q=2$, Table 2 includes the maximum likelihood estimator (MLE) of $\theta$, negative log-likelihood $(-\ell)$ of $\operatorname{MLE}(\theta), D_{k s}$ and associated $p$-value for PQD case. At 0.05 level, Table 2 shows that the generalized FGM model with $p=q=2$ and $\operatorname{MLE}(\theta)=0.464$ is acceptable for the pair $\left(U, C_{s}\right)$ and with $p=q=2$ and $\operatorname{MLE}(\theta)=-0.430$ is acceptable for the pair $(U, L i)$. Now, we test the independence hypothesis against the alternative hypothesis of strict PQD (NQD).

A) For pair of $\left(U, C_{s}\right)$, according to the considered data, we test $H_{0}: \theta=0$ vs $H_{1}: \theta \geq 0.5(\eta=0.5)$ at level $\alpha=0.05$. First, we estimate the distribution functions $F$ and $G$ by their empirical distribution functions and then compute the test statistics mentioned in Section 2. Table 3 includes the values of test statistics, their critical values and associated $p$-values. Table 3 shows that 
Table 4: Values of test statistics, their critical values and associated $p$-values for selected pair $(U, L i)$ in NQD case

\begin{tabular}{|c|c|c|c|c|c|c|c|c|c|c|c|}
\hline & $r_{s}$ & $r_{w}$ & $r_{F y}$ & $r_{e x}$ & LMPR & $\mathrm{CvM}$ & $T$ & $U_{n}(2)$ & $U_{n}(3)$ & $U_{n}(4)$ & $U_{n}(5)$ \\
\hline Test statistic & -0.339 & -0.266 & -0.250 & -0.313 & -0.093 & 0.079 & 0.066 & 0.374 & 0.171 & 0.092 & 0.055 \\
\hline Critical point & -0.234 & -0.234 & -0.234 & -0.234 & -0.094 & 0.056 & 0.028 & 0.419 & 0.240 & 0.150 & 0.096 \\
\hline$p$-value & 0.008 & 0.031 & 0.039 & 0.014 & 0.052 & 0.013 & 0.138 & 0.003 & 0.002 & 0.004 & 0.010 \\
\hline
\end{tabular}

NQD = negative quadrant dependence, LMPR = locally most powerful rank test, CvM = Cramér-von Mises test.

- based on LMPR test, the CvM test, and Güven and Kotz test $(T)$, we cannot reject $H_{0}$ in favor of $H_{1}$.

- the maximum $p$-value is achieved for $T$.

- according to Table 2, the results of Table 3 based on $T$ is coincident with the truth since the data follows generalized FGM with $p=q=2$ and $\theta=0.464$.

Notice that according to Table $1, T$ is the best test statistic for $n=50$ in PQD case.

B) For pair of $(U, L i)$, according to the considered data, we test $H_{0}: \theta=0$ vs $H_{2}: \theta \leq-0.5$ $(\eta=-0.5)$ at level $\alpha=0.05$. Table 4 includes the values of test statistics, their critical values and associated $p$-values. Table 4 shows that

- LMPR test and Güven and Kotz test $(T)$ do not reject $H_{0}$ in favor of $H_{1}$.

- the maximum $p$-value is achieved for $T$.

- according to Table 2, the results of Table 4 based on $T$ is true since the data follows generalized FGM with $p=q=2$ and $\theta=-0.430$.

Notice that according to Table $1, T$ is the best test statistic for $n=50$ in NQD case.

\section{Concluding remarks}

Amini et al. (2010, 2011) compared the power of tests proposed by Kochar and Gupta (1987) in FGM distribution with those proposed by Güven and Kotz (2008) for testing independence against strict PQD or NQD. Some authors introduced a test statistic for testing independence against strict PQD or NQD; however, these tests have not been compared. So selecting the best test and improving the results obtained by Amini et al. (2010, 2011), we consider some rank tests and compare them with the class of tests proposed by Kochar and Gupta (1987) and the test statistic proposed by Güven and Kotz (2008) for testing independence against strict PQD or NQD in generalized FGM family.

In Table 1 and Figures 1-6, we can see that for all $n$ and $\theta$, the test statistic proposed by Güven and Kotz (2008) is the best competitor. These results confirm the results of Amini et al. (2010, 2011). Finally, we explore the results using two actual example for PQD and NQD cases for more justifications.

It is noted that the present results are valid for FGM distribution family and its generalized version introduced by Bairamov and Kotz (2002); therefore, the extension of the current work to other kinds of copulas remains an open problem for new researches.

\section{Acknowledgements}

The research was supported by a grant from Ferdowsi University of Mashhad (No. MS93322JAB). 


\section{References}

Amini M, Jabbari H, Mohtashami Borzadaran GR, and Azadbakhsh M (2010). Power comparison of independence test for the Farlie-Gumbel-Morgenstern family, Communications of the Korean Statistical Society, 17, 493-505.

Amini M, Jabbari H, and Mohtashami Borzadaran GR (2011). Aspects of dependence in generalized Farlie-Gumbel-Morgenstern distributions, Communications in Statistics - Simulation and Computation, 40, 1192-1205.

Bairamov I and Kotz S (2002). Dependence structure and symmetry of Huang-Kotz FGM distributions and their extensions, Metrika, 56, 55-72.

Baker R (2008). An order-statistics-based method for constructing multivariate distributions with fixed marginals, Journal of Multivariate Analysis, 99, 2312-2327.

Cook RD and Johnson ME (1986). Generalized Burr-Pareto-logistic distributions with applications to a uranium exploration data set, Technometrics, 28, 123-131.

Deheuvels P (1981). An asymptotic decomposition for multivariate distribution-free tests of independence, Journal of Multivariate Analysis, 11, 102-113.

Dou X, Kuriki S, Lin GD, and Richards D (2016). EM algorithms for estimating the Bernstein copula, Computational Statistics \& Data Analysis, 93, 228-245.

Farlie DJG (1960). The performance of some correlation coefficients for a general bivariate distribution, Biometrika, 47, 307-323.

Fasano G and Franceschini A (1987). A multidimensional version of the Kolmogorov-Smirnov test, Monthly Notices of the Royal Astronomical Society, 225, 155-170.

Genest C, Quessy JF, and Rémillard B (2006). Local efficiency of a Cramér-von Mises test of independence, Journal of Multivariate Analysis, 97, 274-294.

Genest C and Rémillard B (2004). Test of independence and randomness based on the empirical copula process, Test, 13, 335-369.

Genest C and Verret F (2005). Locally most powerful rank tests of independence for copula models, Journal of Nonparametric Statistics, 17, 521-539.

Gumbel EJ (1960). Bivariate exponential distributions, Journal of the American Statistical Association, 55, 698-707.

Güven B and Kotz S (2008). Test of independence for generalized Farlie-Gumbel-Morgenstern distributions, Journal of Computational and Applied Mathematics, 212, 102-111.

Hájek J and Šidák Ž (1967). Theory of Rank Tests, Academic Press, San Diego, CA.

Hlubinka D and Kotz S (2010). The generalized FGM distribution and its application to stereology of extremes, Applications of Mathematics, 55, 495-512.

Huang JS and Kotz S (1999). Modifications the Farlie-Gumbel-Morgenstern distributions: a tough hill to climb, Metrika, 49, 135-145.

Jung YS, Kim JM, and Kim J (2008). New approach of directional dependence in exchange markets using generalized FGM copula function, Communications in Statistics - Simulation and Computation, 37, 772-788.

Kendall MG and Gibbons JD (1990). Rank Correlation Methods (5th ed), Oxford University Press, New York.

Kochar SC and Gupta RP (1987). Competitors of Kendall-tau test for testing independence against positive quadrant dependence, Biometrika, 74, 664-666.

Kochar SC and Gupta RP (1990). Distribution-free tests based on sub-sample extrema for testing against positive dependence, Australian Journal of Statistics, 32, 45-51. 
Lehmann EL (1966). Some concepts of dependence, Annals of Mathematics and Statistics, 37, 11371153.

Morgenstern D (1956). Einfache beispiele zweidimensionaler verteilungen, Mitteilungsblatt für Mathematische Statistik, 8, 234-235.

Nelsen RB (2006). An Introduction to Copulas (2nd ed), Springer, New York.

Peacock JA (1983). Two-dimensional goodness-of-fit testing in astronomy, Monthly Notices of the Royal Astronomical Society, 202, 615-627.

Rödel E and Kössler W (2004). Linear rank tests for independence in bivariate distributions: power comparisons by simulation, Computational Statistics \& Data Analysis, 46, 645-660.

Serfling RJ (1980). Approximations Theorems of Mathematical Statistics, John Wiley \& Sons, New York.

Shetty ID and Pandit PV (2003). Distribution-free tests for independence against positive quadrant dependence: a generalization, Statistical Methods and Applications, 12, 5-17.

Sklar A (1959). Fonctions de repartition à n dimensions et leurs marges, Publications de l'institue de Statistique de l'Universitte de Paris, 8, 229-231.

Received March 1, 2016; Revised May 2, 2016; Accepted May 3, 2016 